Proceedings of the 42th "Jaszowiec" International School and Conference on the Physics of Semiconductors, Wisła 2013

\title{
Octagonal Defects as the Source of Gap States in Graphene Semiconducting Structures
}

\author{
M. PelC ${ }^{a}$, W. JASKÓlski ${ }^{a}$, A. AyUela ${ }^{b}$ AND L. ChiCO ${ }^{c}$ \\ ${ }^{a}$ Institute of Physics, Faculty of Physics, Astronomy and Informatics, Nicolaus Copernicus University \\ Grudziądzka 5, 87-100 Toruń, Poland \\ ${ }^{b}$ Centro de Física de Materiales CSIC-UPV/EHU, Donostia International Physics Center \\ 20018 San Sebastián, Spain \\ ${ }^{c}$ Instituto de Ciencia de Materiales de Madrid, CSIC, C/Sor Juana Inés de la Cruz 3, 28049 Madrid, Spain \\ We study graphene nanoribbons and carbon nanotubes with divacancies, i.e., local defects composed of one \\ octagon and a pair of pentagons. We show that the presence of divacancies leads to the appearance of gap states, \\ which may act as acceptor or donor states. We explain the origin of those defect-localized states and prove that \\ they are directly related to the zero-energy states of carbon ring forming the octagonal topological defect.
}

DOI: 10.12693/APhysPolA.124.777

PACS: 73.22.Pr, 61.72.Qq

\section{Introduction}

Different types of structural defects, like mono- and multi-vacancies or hexagonal lattice reconstructions, may strongly influence and modify the electronic, magnetic and transport properties of the graphene nanostructures [1-4]. They have been experimentally observed in graphene-based systems $[5,6]$. Such defects may naturally appear during the growth process or can be on purpose created by electron/ion irradiation [7]. Pentagon and heptagon topological defects can appear at junctions between carbon tubes during their growth [8]. Octagonal defects can occur at T- or Y-junctions as well as at junctions between tubes of dissimilar radii [9-11]. It has been recently reported that octagonal defects may form linear grain boundaries in graphene, behaving as one-dimensional metallic wires [12]. This is because they reveal defect-localized flat bands at the Fermi energy $\left(E_{\mathrm{F}}\right)$, which in some cases may lead to spontaneous magnetization $[1,2,13]$.

We concentrate on isolated octagonal defects, which most commonly appear at reconstructed divacancies, where they are accompanied by pairs of pentagons (5-8-5 defect) as shown in Fig. 1a. A direct observation of such structures using transmission electron microscopy has been reported in Refs. [14, 15]. Their stability and role on transport properties in defected graphene have been also theoretically investigated $[6,16]$. So far, most investigations were mainly focused on gapless systems, like zigzag nanoribbons and metallic carbon tubes [17].

In this paper we explore their influence on the electronic properties of semiconducting graphene nanoribbons and carbon nanotubes. We show that such defects are the source of localized states in the energy gap, acting as acceptor or donor states. Most importantly, we explain the nature and origin of the gap states localized at divacancies. We prove that they appear because of the very specific energy spectrum of the octagonal carbon ring. We investigate also how the energies of the

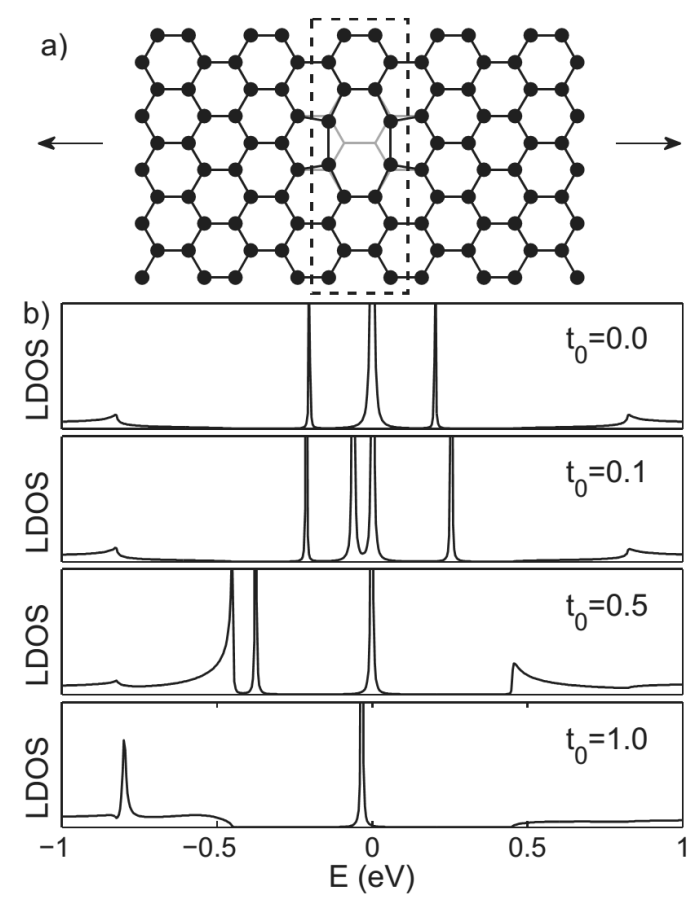

Fig. 1. An armchair nanoribbon of width $N=10$ with a 5-8-5 defect: (a) geometrical structure of the ribbon with the defect; the dashed rectangle indicates the region where the LDOS is calculated; (b) LDOS for different values of the hopping parameter between the octagonal ring and the neighbouring atoms: $t_{0}=$ $0,0.1,0.5,1$ (in units of $t$ ).

octagon-localized states depend on the defect orientation versus the ribbon or tube axis.

\section{Model and method}

We focus on single divacancies reconstructed into 5-8-5 defects in the semiconducting graphene nanoribbons and 
carbon nanotubes. We choose armchair ribbons, as they have no edge-localized states that could mix with the defect-localized state [13]. The width of the investigated ribbons and the diameter of the nanotubes are chosen to be rather small, in order to obtain systems with wide energy gaps. We also perform calculations for superlattices (SL), where the defect periodically appears in each unit cell.

We perform calculations within the $\pi$-electron tight binding (TB) approximation with hopping parameter $t=-2.7 \mathrm{eV}$. We have checked that changes in $t$ induced by the defect cause negligible changes in the calculated energy spectra, in agreement with previous calculations $[1,8]$. For non-periodic systems, like a single defect in a perfect ribbon, we use the Green function matching technique $[18,19]$ to calculate the local density of states (LDOS). For the superlattices, the TB Hamiltonian is directly diagonalized, yielding the band structures and the corresponding wave functions.

To investigate the origin and the nature of localized gap states, we disconnect the octagonal defect by setting the hopping parameter $t_{0}$ between the defect and the neighbour atoms equal to zero. As the result, a void is left in the graphene lattice. Next, we gradually reconnect the octagonal defect, by increasing $t_{0}$ up to 1 (in $t$ units), and we analyze the changes in the LDOS.

\section{Results}

First, we consider an infinite narrow graphene ribbon with a single 5-8-5 defect, as shown in Fig. 1a. The width $N$ of the ribbon is defined following Ref. [20] by counting the carbon dimers across it. The $N=10$ defect-free armchair ribbon is a semiconductor with an energy gap of almost $1 \mathrm{eV}$. We first separate the octagonal ring from the 5-8-5 defect by setting $t_{0}$ equal to zero. After disconnecting the octagon we get the ribbon with a void composed of eight nodes, each one having a single dangling bond. The corresponding LDOS, calculated in the region enclosed by a dashed rectangle in Fig. 1a, is shown in the first part in Fig. 1b. Comparing to the LDOS of the defect-free ribbon, the gap between energy continua increases negligibly, but now three peaks appear in it: one peak at $E=0$ and two peaks at $E= \pm 0.208 \mathrm{eV}$. The $E=0$ state is doubly degenerate and belongs to the disconnected octagonal ring. This is due to the fact that an isolated octagonal carbon-atom ring has two zero energy states. The states at $E= \pm 0.208 \mathrm{eV}$ are also localized, their wave functions locate at the edge of the void. This localization is related to the zigzag shape of the internal void edge. Both states are mostly situated at the edge of the void. As there are no odd-numbered carbon rings mixing the two sublattices, the electron-hole symmetry is preserved [13].

When we gradually reconnect the octagon by increasing the value of $t_{0}$, the LDOS peaks related to the void-localized states spread away and merge the band continua. This is shown in Fig. 1b. The degenerate $E=0$ peak, which originates from the pure octagonal ring, splits into two. One peak moves away to the negative bulk continuum, while the second stays close to $E=0$. Finally, for $t_{0}=1$, when the defect is fully connected, we obtain one gap state with $E=-0.036 \mathrm{eV}$, mostly localized at the octagon.

To see the exact localization of this state, we consider the superlattice ribbon with a very long unit cell, each containing the 5-8-5 defect inside. Figure 2a shows its band structure. There is a dispersionless band with $E=-0.0321 \mathrm{eV}$ in the main energy gap. The corresponding TB wave function (for the wave vector $k=0$ ) is shown in Fig. 2c. The size of the dot reflects the value of the function on the particular node; empty and filled dots denote opposite signs of the function. It is mostly localized at the defect and decays away from it.

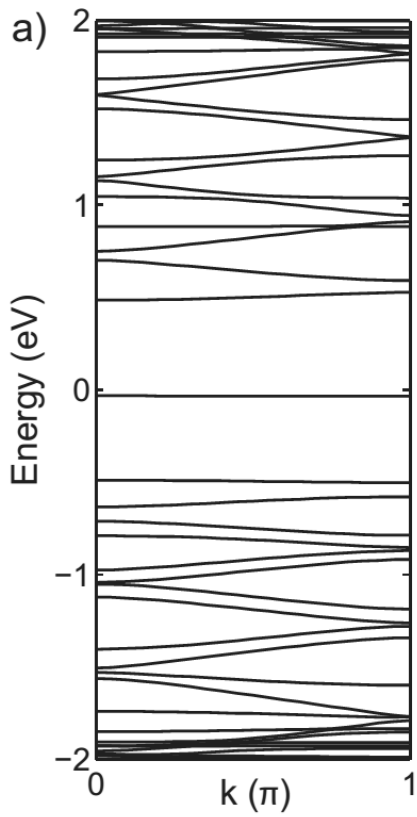

b)
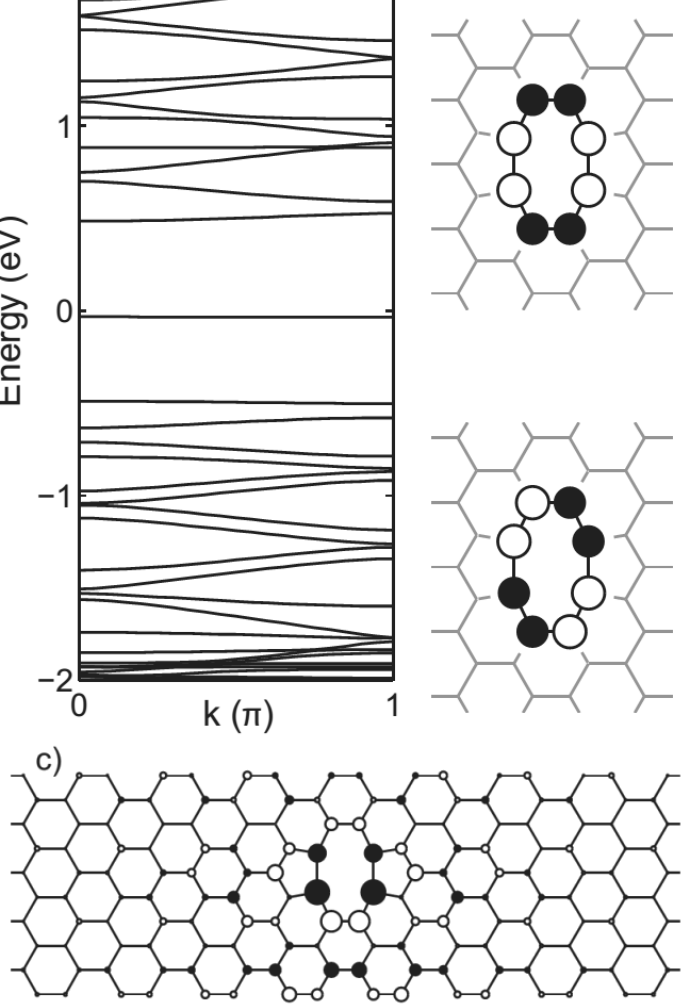

Fig. 2. (a) Band structure of nanoribbon superlattice $\left(t_{0}=1\right)$; (b) TB wave functions of the $E=0$ states in the isolated octagonal ring. Dashed grey lines show the hexagonal atomic structure of the ribbon around the octagon when it is connected to the lattice. (c) TB wave function of the superlattice gap state with $E=$ $-0.0321 \mathrm{eV}$.

Attaching the octagon to the ribbon causes the appearance of pentagons, with the subsequent sublattice mixing which breaks electron-hole symmetry. Thus, the octagon-localized state moves slightly away from $E=0$. In the case of SL the Fermi level $\left(E_{\mathrm{F}}\right)$ is below the energy 
of the gap state (band), which is therefore unoccupied and acts as deep acceptor state.

To explain the observed difference in the behavior of the two $E=0$ states of the octagonal ring when it is attached to the void, let us recall the condition for the existence of $E=0$ states in the tight-binding approximation: the sum of the wave function coefficients on the neighbouring nodes has to be equal to zero for every node in the system $[13,20]$. The two $E=0$ states of the isolated octagonal ring are shown in Fig. 2b*. When the octagon is connected to the void, two pentagons are created. The state that has the same sign of the function on the two nodes shared between the pentagon and the octagon, fulfills this condition approximately. For a very wide ribbon this condition would be almost perfectly satisfied. The condition for the second state, i.e., the one with different signs of function on the shared nodes, is strongly disturbed by the pentagons. Therefore, the zero-sum condition cannot be fulfilled and the second state moves away from $E=0$.

We have also performed calculations for wider ribbons, with similar results. Wider ribbons have narrower energy gaps, but one defect-localized state always appears in the middle of the gap, i.e., close to $E=0$. Furthermore, we have investigated symmetric ribbons, i.e., those with a mirror plane along the ribbon. In such a case, the octagon-localized gap state has an energy closer to the valence band edge and plays the role of shallow acceptor.

\subsection{Divacancies in carbon nanotubes}

Here we present the results of calculations performed for the zigzag nanotube $(5,0)$, formed by rolling up an $N=10$ armchair nanoribbon. The defect-free nanotube is a semiconductor with a wide energy gap of $\approx 2 \mathrm{eV}$. When a single 5-8-5 defect is introduced, two localized states appear in the energy gap.

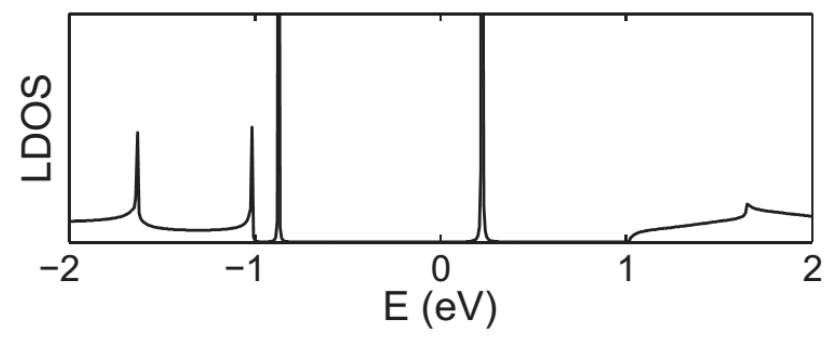

Fig. 3. LDOS of the $(5,0)$ zigzag nanotube (a rolled-up $N=10$ armchair ribbon) with the 5-8-5 defect. The Fermi level is between the gap states.

The calculated LDOS is shown in Fig. 3. One of these discrete energy levels appears close to the middle of the gap, at $E=0.261 \mathrm{eV}$, and the second one closer to the

\footnotetext{
*These are two possible realizations of the zero-energy-state wavefunctions of an isolated octagonal carbon ring
}

bulk continuum at $E=-0.872 \mathrm{eV}$. Both of them originate from the $E=0$ states of the isolated octagonal ring; both are localized at the defect, although the upper energy state is mostly located at the defect, while the lower one spreads far away from it. Thus, contrary to the previous cases, now the second peak does not reach the continuum, since the energy gap is twice wider than the $N=10$ ribbon case. The Fermi level is between the two gap states. Therefore, they play the role of an occupied acceptor state (the lower one) and an unoccupied donor state (the upper one).

\subsection{Orientation dependence}

In this subsection we check how the energies of the gap states depend on the orientation of the defect. We rotate the whole 5-8-5 defect by an angle of $\frac{\pi}{3}$. A schematic picture of a ribbon with the rotated defect is shown in Fig. 4a. The LDOS shown in Fig. 4b presents two gap states: one at $E=0.182 \mathrm{eV}$ and another one close to the valence band continuum, at $E=-0.399 \mathrm{eV}$. Both originate from the octagon. The first state is mostly localized at the defect, while the second one spreads far away from it. The Fermi level is between these two peaks. The character of these gap states is the same as in the case of a non-rotated divacancy in a carbon nanotube (see previous subsection).

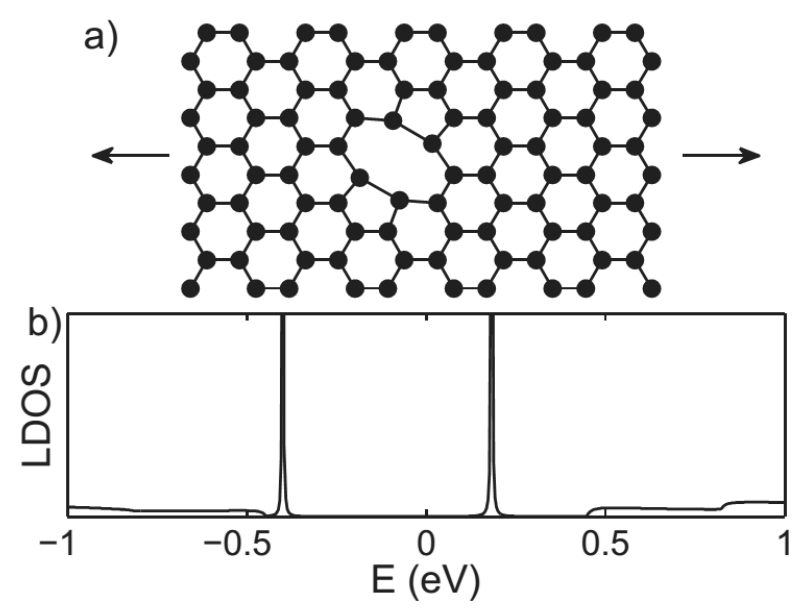

Fig. 4. (a) Geometry and (b) LDOS of the $N=10$ armchair nanoribbon with an 5-8-5 defect rotated by $\frac{\pi}{3}$. The Fermi level is between the gap states.

\subsection{Two defects}

Finally, we consider a ribbon with two 5-8-5 defects, but one of them rotated by $\frac{\pi}{3}$. Its geometry is shown in Fig. 5a. The LDOS, presented in Fig. 5b, shows three peaks in the primary energy gap: at $E=-0.376 \mathrm{eV}, E=$ $-0.14 \mathrm{eV}$ and $E=0.3 \mathrm{eV}$. All of them originate from the $E=0$ states of both octagons. The energies of the two upper states are close to the energies of the gap states of ribbons with single defects, i.e., the unrotated defect case $(E=-0.036 \mathrm{eV})$ and the rotated one $(E=0.182 \mathrm{eV})$. If 
we increase the distance between defects, their energies get closer to the typical values for single-defect systems, shown in Figs. 1 and 4.
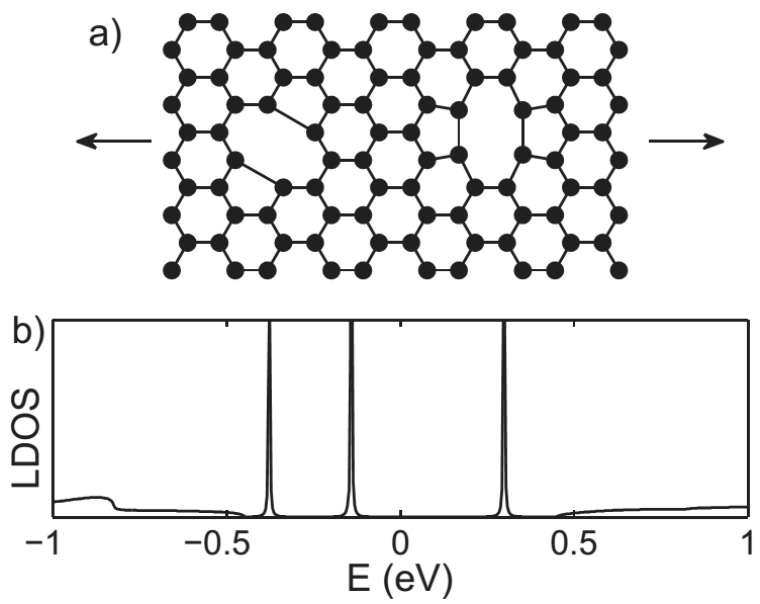

Fig. 5. (a) Schematic atomic structure and (b) LDOS of an $N=10$ armchair nanoribbon with two 5-8-5 defects, one of them rotated by $\frac{\pi}{3}$.

\section{Conclusions}

We have investigated the influence of divacancies on the energy spectra of semiconducting graphene nanoribbons and carbon nanotubes. We have shown that such defects are the source of gap-localized states, which may act as deep or shallow acceptor states or as unoccupied donor states, depending on their energy. We have found that the appearance of divacancy-localized gap states is directly related to the energy spectrum of the separated octagonal carbon ring. Such ring, cut out from the system, has a doubly degenerate zero-energy level. When the octagonal ring is attached to the graphene lattice, only one of these pair of states can approximately fulfill the condition necessary to the existence of midgap $E=0$ states. This midgap state is the one which remains as a characteristic divacancy-localized state when the octagon is attached to the lattice forming the 5-8-5 defect. This is in agreement with our previous finding concerning the zero-energy state localization at octagons which occurs in strongly curved graphene structures [11].

Divacancy defects can be intentionally created in graphene structures. Therefore, the knowledge on the conditions under which they can play a role of acceptor or donor impurities may be useful for future nanoelectronic devices based on graphene.

\section{Acknowledgments}

This work was supported by the Polish National Science Center (grant DEC-2011/03/B/ST3/00091), the Spanish Ministry of Economy and Competitiveness (grant No. FIS2012-33521), the Basque Departamento de Educación and the University of the Basque Country (grant No. IT-366-07), the Basque Departamento de Industria and the Diputación Foral de Guipuzcoa under the ETORTEK program (NANO-IKER grant No. IE11$-304)$.

\section{References}

[1] M. Pelc, L. Chico, A. Ayuela, W. Jaskólski, Phys. Rev. B 87, 165427 (2013).

[2] W. Jaskólski, M. Pelc, L. Chico, A. Ayuela, IEEE Conf. Nanotechnol. 1, 1 (2012).

[3] R.R. Nair, M. Sepioni, I-Ling Tsai, O. Lehtinen, J. Keinonen, A.V. Krasheninnikov, T. Thomson, A.K. Geim, I.V. Grigorieva, Nature Phys. 8, 199 (2012); S. Kattel, P. Atanassov, B. Kiefer, J. Phys. Chem. C 116, 8161 (2012).

[4] A. Lherbier, S.M.-M. Dubois, X. Declerck, Y.-M. Niquet, S. Roche, J.-C. Charlier, Phys. Rev. B 86, 075402 (2012).

[5] J.M. Carlsson, M. Scheffler, Phys. Rev. Lett. 96 046806 (2006); M.M. Ugeda, I. Brihuega, F. Guinea, J.M. Gómez-Rodríguez, Phys. Rev. Lett. 104. 096804 (2010); A. Ayuela, L. Chico, W. Jaskólski, Phys. Rev. B 77, 085435 (2008).

[6] M.M. Ugeda, I. Brihuega, F. Hiebel, P. Mallet, J.-Y. Veuillen, J.M. Gomez-Rodriguez, F. Yndurain, Phys. Rev. B 85, 121402(R) (2012).

[7] A. Hashimoto, K. Suenaga, A. Gloter, K. Urita, S. Iijima, Nature (London) 430, 870 (2004).

[8] L. Chico, V.H. Crespi, L.X. Benedict, S.G. Louie, M.L. Cohen, Phys. Rev. Lett. 76, 971 (1996).

[9] M. Menon, D. Srivastava, Phys. Rev. Lett. 79, 4453 (1997).

[10] M. Terrones, F. Banhart, N. Grobert, J.-C. Charlier, H. Terrones, P.M. Ajayan, Phys. Rev. Lett. 89 , 075505 (2002).

[11] W. Jaskólski, M. Pelc, H. Santos, L. Chico, A. Ayuela, Phys. Status Solidi C 7, 2 (2010).

[12] J. Lahiri, Y. Lin, P. Bozkurt, I.I. Oleynik, M. Batzil, Nature Nanotech. 5, 325 (2010).

[13] W. Jaskólski, A. Ayuela, M. Pelc, H. Santos, L. Chico, Phys. Rev. B 83, 235424 (2011).

[14] A.W. Robertson, C.S. Allen, Y.A. Wu, K. He, J.N Olivier, A.I. Kirkland, J.H. Warner, Nature Commun. 3, 1144 (2012).

[15] J. Kotakoski, A.V. Krasheninnikov, U. Kaiser, J.C. Meyer, Phys. Rev. Lett. 106, 105505 (2011).

[16] Y. Kim, J. Ihm, E. Yoon, G.-D. Lee, Phys. Rev. B 84, 075445 (2011).

[17] R.Y. Oeiras, F.M. Araújo-Moreira, E.Z. da Silva, Phys. Rev. B 80, 073405 (2009); F. Hu, J. Zhou, J. Appl. Phys. 110, 113702 (2011).

[18] M.B. Nardelli, Phys. Rev. B 60, 7828 (1999).

[19] L. Chico, L.X. Benedict, S.G. Louie, M.L. Cohen, Phys. Rev. B 54, 2600 (1996).

[20] K. Nakada, M. Fujita, G. Dresselhaus, M.S. Dresselhaus, Phys. Rev. B 54, 17954 (1996). 